DOI: $10.17516 / 1997-1397-2020-13-5-631-643$

УДК 532.526

\title{
Experimental and Numerical Study of Free Convection Heat Transfer Around the Junction of Circular Cylinder and Heated Vertical Plate
}

\author{
Hamid Malah* \\ Yurii S. Chumakov ${ }^{\dagger}$ \\ Peter the Great St. Petersburg Polytechnic University \\ St. Petersburg, Russian Federation
}

Received 10.06.2020, received in revised form 21.07.20, accepted 23.08.2020

\begin{abstract}
The present study investigates the effects of a circular cylinder on the three-dimensional characteristics of free convective heat transfer. The circular cylinder is mounted horizontally on a heated vertical plate and is categorized as high aspect ratio obstacle, which means the height of cylinder is comparable to its diameter. The obtained results are provided for the laminar flow regime. In addition, during numerical study the governing differential equations are solved around the Grashof number equals to $3 \times 10^{8}$. In order to illustrated the regions of high gradients of temperature, the flow temperature is shown in terms of non-dimensional contours and diagrams. At the near junction region in upstream of cylinder, by description of heat transfer coefficients represented to the temperature gradients at intended points, the effects of cylinder emplacement on the heat transfer rate is surveyed. As expected, the value of the buoyancy-induced heat transfer coefficient increases at the cylinder junction in the upstream side. The maximum value of heat transfer coefficient is seen at the symmetry plane of study domain, which is corresponded to the location of horseshoe vortex system core. Finally, by deviation calculating between numerical and experimental results also by analysis of the experimental method uncertainty the validity and reliability of numerical and experimental approaches are proved.
\end{abstract}

Keywords: juncture flow, free convection heat transfer, laminar boundary layer, heat transfer coefficient.

Citation: H. Malah, Y.S. Chumakov, Experimental and Numerical Study of Free Convection Heat Transfer Around the Junction of Circular Cylinder and Heated Vertical Plate, J. Sib. Fed. Univ. Math. Phys., 2020, 13(5), 631-643. DOI: 10.17516/1997-1397-2020-13-5-631-643.

\section{Introduction}

Free convection flows around surface-mounted isothermal circular cylinders are found in many industrial and environmental applications. Flow through bluff body located on a heated plate is complex and can contribute to significant non-uniformities of local heat transfer [1]. Such flows can attributed to the body junction of different heat exchanger units and cooling systems. In most industrial applications, the focus is on heat transfer and factor that governs the flow pattern. Typical geometry of such applications can be represented, as a circular cylinder mounted on heated vertical plate with a free convection boundary layer developing along it. A large

\footnotetext{
*hamid.malah@gmail.com

†chymakov@yahoo.com

(C) Siberian Federal University. All rights reserved
} 
adverse pressure gradient in the encounter region where the free convection boundary layer approaches the circular cylinder leads to separate the boundary layer in front of the stagnation point. Development of such flows depends both on intensity of the separation and on the arising buoyancy force acting on the heated flow pushed away from the heated plate. In addition, flow structure forming depends on bluff body shape and its aspect ratio. Compared to the flow past a short obstacle, the flow past a high aspect ratio bluff body placed on the heated vertical plate is more complex due to the effects of the plate and free convection boundary layer [2].

Experimental and computational studies of free convection flows over a bluff body have been reported in the several literature [3-5], that all confirming the anticipated increase in heat transfer in the stagnation region in front of the bluff body and its decrease in the wake region. Heat transfer from simple plate or around single cylinder intensively differs from a complex system consist of bluff body placed on the plate due to effects of boundary layer arising from the plate leading edge on bluff body in the encounter region. Despite unconfined cylinders or bluff body is placed on the horizontal plate [6,7], the free convection heat transfer around a complex system included high aspect ratio circular cylinder mounted on heated vertical plate in laminar regime is not well understood. The better understanding of this complex system is critically important because of its practical applications. In the case of bluff body mounted on heated plate, there are some papers, which present the analysis of free convection heat transfer at the junction of low aspect ratio isothermal cylinder mounted on a heated wall $[2,8]$. These papers $[2,8]$ show that the low aspect ratio bluff body which completely embedded in free convection boundary layer leads to heat transfer enhancement.

The present paper covers numerical results of time-based computations, which categorize the effects of high aspect ratio cylinder mounting on free convection heat transfer at the junction of the circular cylinder and heated vertical plate with approaching laminar free convection boundary layer. The axis of the circular cylinder directed normally to the plate. The study considers the case, which an adiabatic circular cylinder obviously crosses from thermal boundary layer arisen on the vertical plate, which means the cylinder height $(H)$ is comparable to the diameter of cylinder $(D)$. In order to confirm obtained computational results in upstream region of cylinder, the experimental data for the same problem are presented in parallel.

\section{Problem definition}

This study is performed by using three-dimensional Navier-Stokes and energy balance equations to solve the problem of laminar flow of a viscous incompressible fluid, which regarded to the Boussinesq approximation over a circular cylinder mounted on a rectangular heated vertical plate. In order to reduce the non-linearity of the governing equations meanwhile the numerical solution, the density variation was neglected in all terms of governing equations except the buoyancy term. Therefore, the following set of equations, which will applied to consider computational domain by finite volume method is in good accuracy with original set of governing equations:

$$
\begin{gathered}
\nabla \cdot V=0, \\
\rho \frac{\partial V}{\partial t}+\rho(V . \nabla) V=\rho \beta g\left(T-T_{\infty}\right)-\nabla P+\mu \nabla^{2} V, \\
\rho C_{p} \frac{\partial T}{\partial t}+\rho C_{P} V . \nabla T=\lambda \nabla^{2} T .
\end{gathered}
$$

The system of equations (1) include: the velocity of a parcel of fluid, $V$; density, $\rho$; time, $t$; volume thermal expansion coefficient, $\beta$; gravitational acceleration, $g$; the temperature of a 
Table 1. Boundary conditions of problem

\begin{tabular}{cc}
\hline Index & Applied conditions \\
\hline 1 & $V_{X}=V_{Y}=V_{Z}=0, \partial P / \partial n=0, \partial T / \partial n=0$ \\
2 & $V_{X}=V_{Y}=V_{Z}=0, \quad \partial P / \partial Z=0, T=T_{W}$ \\
3 & $V_{X}=V_{Y}=V_{Z}=0, \quad \partial P / \partial Z=0, T=T_{\infty}$ \\
4 & $V_{X}=V_{Y}=V_{Z}=0, \quad \partial P / \partial Z=0, T=T_{\infty}$ \\
5 & Symmetry \\
6 & Symmetry \\
7 & $P=P_{\infty}, T=T_{\infty}$ \\
\end{tabular}

parcel of fluid, $T$; surrounding medium temperature, $T_{\infty}$; the local pressure of a parcel of fluid, $P$; dynamic viscosity, $\mu$; specific heat at constant pressure, $C_{P}$ and thermal conductivity, $\lambda$.

\section{Computational aspects}

In this study, for different analyzed cases, the diameter of the cylinder $(D)$ equals to $0.02[\mathrm{~m}]$ and the height of the cylinder $(H)$ equals to $2 D$. The height of cylinder is selected in this way to cross thermal boundary layer entirely. The emplacement position of cylinder is considered to be at the place, where the Grashof number along vertical plate $\left(G r_{y}\right)$ on simply vertical plate without cylinder (undisturbed upward flow), equals to desired value of the laminar Grashof number. The origin of the system of coordinate is placed $31.5 \mathrm{D}$ from the leading edge of the isothermal vertical plate and coincides with the center of circular cylinder cross section at junction region and consequently the approaching flow at the location of cylinder is fully developed laminar flow. The $X$-axis corresponds to the horizontal direction, the $Y$-axis aligns to the vertical direction and the $Z$-axis is normal to the vertical plate. The coordinate system and computational domain, which used in the present study, are shown in Fig. 1. In order to cautiously simulate the characteristics of the flow in the vicinity of the cylinder, the dimensions of computational domain are drawn out $117 D$ in height and $7 D$ width. For the normal direction, the domain size is set to $10 D$, to provide a sufficient wide space for ultimate develop of convective boundary layer thickness $\left(\delta_{0}\right)$ arising from heated vertical plate. In order to avert the effects of the solid boundaries on the free convective flow pattern also on the heat transfer characteristics, the lower face of the computational domain is located at $40.5 \mathrm{D}$ upstream of the cylinder axis and the upper one is placed at $76.5 D$ downstream, as shown in Fig. 1a.

By authors' knowledge, the free convective flow in laminar regime does not depend on initial condition of solution, especially in our case the final solution is independent from given initial condition [2]. In this paper, there is no initial velocity around leading edge of heated vertical plate and at the beginning of calculations (first time step). Therefore, velocity in all directions assumed as zero. By starting numerical procedure $(t=0[s])$, the temperature at the vertical plate is increased suddenly from $T_{\infty}=293.15[K]$ to $T_{W}=333.15[K]$ and maintained at this value. Heat is transferred initially by pure conduction to the surrounding medium and this initial conductive phase is characterized by arisen thermal boundary layer on vertical plate, until a certain critical time is happened when buoyant flow arises and interacts with cylinder surfaces also heated vertical plates and finally leads to forming free convective layer around the solid surfaces.

The boundary conditions are formulated as corresponding mathematical equations as follows in Tab. 1 (see Fig. 1a for numbering of the computational domain boundaries). 
Fig. 1b shows the blocked structure of grid for meshing system, which covers the computational domain by eight-node hexahedron elements. The computational domain is discretized using body-fitted mesh adapted to the geometry of the domain in order to carry out high-resolution numerical simulation. The final quantity of three-dimensional computational grid elements approximately equal to 6.2 million. The grid is clustered to heated vertical plate along $Z$-axis by considering that the elements size in the near-wall regions are about $0.02 D$. In the normal direction to the vertical plate, the grid consists of 150 nodes, where 50 nodes are located inside the thickness of undisturbed boundary layer. The multi-block structured grid is originated from two-dimensional grid of the $X Y$ plane.

In Fig. 1b is shown the numbering of the blocked structure of two-dimensional grid, which is explained in the following. An O-type block (8) embedded in the clustered region close to the cylinder (9) to discretize this enclosing area. The diameter of this O-type block, which is centered at the center of the cylinder equals to $2.1 D$. An additional square block (10) with edges dimension equal to $3 D$, which enclose the O-type mesh area, is embedded to enhance fine grid computation around the cylinder. In the $X Y$ plane, the elements size of grid in the O-type block and square block respectively varied between $0.013-0.027 D$ and $0.023-0.093 D$. Four additional grid blocks (11) are selected between the refined square block around the cylinder and outer blocks (12) with coarse mesh, which served to align the skewness of the forming elements. Finally, six blocks (13) were constructed adjacent of leading and trailing edges of vertical plate, where the grid of these blocks were clustered to the heated vertical plate edges in order to correctly describe arising flow in these critical regions.
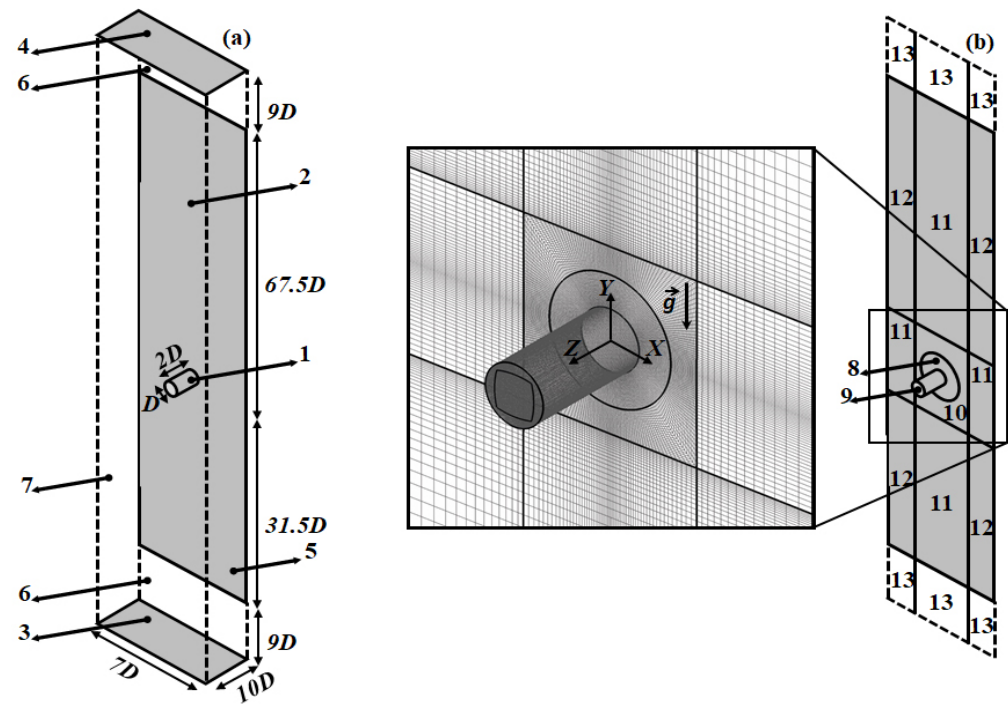

Fig. 1. The schematic representation of the problem configuration: (a) - computational domain, (b) - grid layout

In this work, the commercial package ANSYS-Fluent 16.2 [9] was used to simulate numerically three-dimensional disturbing effects around circular cylinder in the upstream region. To evaluate the effects of computational parameters include time step and mesh sizes during numerical solution, here, four different simulations were performed, which differs from each other in grid refinement and time step. These different configurations were applied to surface-mounted adiabatic circular cylinder on the heated vertical plate. In Tab. 2, two symmetry plane characteristics 
of the horseshoe vortex system include diagonal distance of necklace vortex axis coordinates relative to the circular cylinder axis $\left(L_{P V}\right)$ and in normal direction to the vertical plate $\left(C_{P V}\right)$ were provided to compare. Tab. 2 shows a high degree of fidelity between refined and coarse grids. In addition, Tab. 2 proves the ineffectiveness of reducing physical time step on characteristics of horseshoe vortex system. Furthermore, Tab. 2 was used to validate the numerical simulation method by previous numerical study [2]. The analysis of given values in Tab. 2 confirms the reliability of numerical solutions. As result of reliability analysis, which presented in Tab. 2, the effectiveness of applied numerical configuration in ANSYS-Fluent 16.2, is emphasized. Therefore, the time step used in the present computation was, in general, at $0.002[s]$ and flow parameters are stabilized with an accuracy equal to $10^{-6}$ at physical time approximately equal to $200[s]$.

Table 2. Computational parameters effects on the necklace vortex characteristics

\begin{tabular}{ccccc}
\hline Index & Number of cells & Time step $[\mathbf{s}]$ & $\mathbf{L}_{\mathbf{P V}}[\mathbf{m}]$ & $\mathbf{C}_{\mathbf{P V}}[\mathbf{m}]$ \\
\hline Coarse & 6200000 & 0.001 & 0.0128 & 0.0024 \\
Coarse & 6200000 & 0.002 & 0.0128 & 0.0024 \\
Refined & 20500000 & 0.001 & 0.0129 & 0.0024 \\
Refined & 20500000 & 0.002 & 0.0129 & 0.0024 \\
[2] & 3650000 & Steady State & 0.0135 & 0.0022 \\
\hline
\end{tabular}

\section{Experimental investigation}

The difficulty in the experimental study of free convection flows compared with forced flows is due to the complexity of providing experimental apparatus, which can generate stable free convection flow with determined parameters. In this study, free convective boundary layer near a heated vertical plate is provided, by using an experimental setup described in experiments of other authors [10]. Here the characteristics of the used experimental apparatus allow simulating different flow regimes, up to the Grashof number equal $5 \times 10^{11}$.

The main part of this experimental apparatus is the junction of cylinder and heated vertical plate. A solid polymer circular cylinder is mounted on vertical plate using light glue. The diameter of cylinder fixed to $0.02[\mathrm{~m}]$ and its height is equal $2 \mathrm{D}$. The adiabatic condition on cylinder surfaces is provided by very low thermal conductivity of polymer. The vertical plate is made of duralumin sheet with dimensions of $250 D \times 44 D \times 0.02 D$, and its working surface, along which free convection flow arises, is polished.

The temperature of studying domain is measured using a resistance thermometer sensor, which is controlled remotely by computer. During the experimental investigation, the surrounding medium temperature varies between 293.15 and $295.15[K]$. Since it is not possible to measure the temperature of vertical plate $\left(T_{W}\right)$ by resistance thermometer sensor precisely, the wall temperature is approximated by linearization of temperature profiles on desired points.

\section{Results and discussion}

In this part, the results of the numerical simulation in comparison with experimental data for a high aspect ratio circular cylinder $(H / D=2)$, which crosses the formed boundary layer entirely, are presented. It is noteworthy that the thermal boundary layer thickness $(\delta)$ assumed as the $Z$ coordinate where the local temperature differs from the surrounding medium temperature less than two percent. Here, the computed thickness of developed thermal boundary layer along 
vertical plate at emplacement of cylinder $(Y / D=0)$ equals $0.5 H$, which ensures that cylinder height entirely crosses the formed boundary layer. In this case, the free convection heat transfer is controlled by the intensity of buoyancy force, which depends on the local temperature difference between heated vertical plate and surrounding medium $\left(T_{W}-T_{\infty}\right)$ [2]. Subsequently in order to study free convection heat transfer parameters at first, the temperature field is illustrated by using dimensionless temperature. In continue, by demonstrating dimensionless temperature profiles at different $Y Z$ sections $(X / D=0,0.6,0.7,0.85)$, the free convection heat transfer coefficient $(\alpha)$ is investigated in both numerical and experimental study to realize the effects of cylinder emplacement on the flow configuration in the upstream region of the cylinder. In this paper, numerical and experimental results of temperature fields and heat transfer rate were obtained for laminar Grashof number equals to $3 \times 10^{8}$. The extremely high Grashof number leads to apply enormous time steps for numerical solution procedure also leads to convergence problems. Furthermore, the provided experimental study demonstrated that transient regime is occurred only after $45 \mathrm{D}$ from leading edge of the heated vertical plate. Therefore, the characteristics of laminar regime approximately extend to Grashof number equals $5 \times 10^{9}$. Thus, the Grashof number was selected in this way to satisfy the requirements of the practical applications by considering the numerical limitations. To sum up, by comparing the numerical results to experimental data the validity of the computational simulation and their underlying assumptions and simplifications are verified.

Since the temperature of the vertical plate and surrounding medium in numerical simulation are given as isothermal temperature, and in experimental investigation is measured by thermal sensors, there is dissimilarity among these values in numerical and experimental study. Thus, the obtained values from the experimental investigation cannot be directly compared to the computed values. To combat this problem, in present work, a dimensionless temperature $\left(T^{+}\right)$ is defined as follows:

$$
T^{+}=\frac{T_{W}-T}{T_{W}-T_{\infty}}
$$

Which is determined as the ratio of the temperature difference between vertical plate and desired point of investigated ambient $\left(T_{W}-T\right)$, to the temperature difference between vertical plate and surrounding medium $\left(T_{W}-T_{\infty}\right)$. As stated in previous parts, the temperature of surrounding medium $\left(T_{\infty}\right)$ in numerical simulation considered as $293.15[K]$ and in experimental study varies between $293.15[K]$ to $295.15[K]$. In addition, the temperature of vertical plate in numerical solution fixed on $333.15[K]$ and in experimental investigation is obtained by linearization of temperature profile on desired point.

The dimensionless temperature field comparison between the numerical solution and experimental investigation is shown in Fig. 2 for different $Y Z$ planes around the junction of cylinder and heated vertical plate. The present experimental results qualitatively are in good agreement with their numerical results at different $Y Z$ planes. The obtained temperature field in upstream region of the cylinder is symmetric. It is especially noted here that the existence of step-like structure in Fig. 2 for experimental temperature field is due to spatial step among gathered experimental data. Subsequently when the contour of temperature is drawn by using the triangulation of data method in modeling software, there are depicted step-like structure. Furthermore, the white regions in Fig. 2a and Fig. 2b correspond to very closed cylinder surfaces regions, which could not be considered due to the limitation of experimental measurement.

As seen in Fig. 2, the buoyancy-induced flow develops on the vertical plate due to temperature gradient between the heated vertical plate and the cold ambient fluid. This gradient leads to 
(a)- Experimental, $X / D=0$

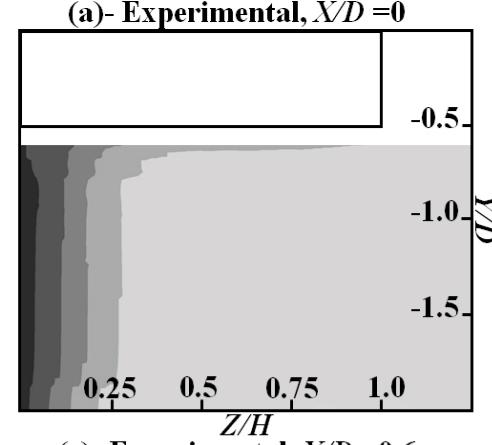

(c)- Experimental, $X / D=0.6$

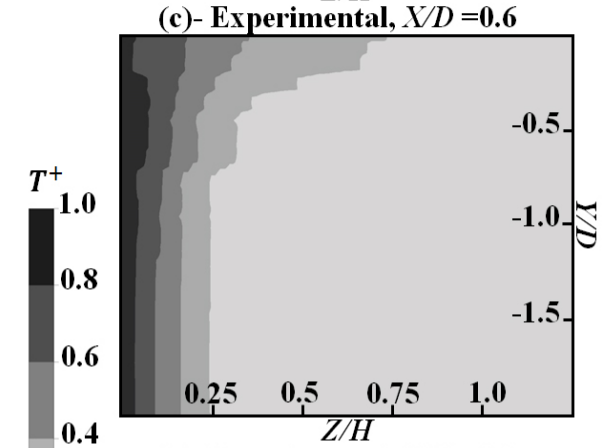

(e)- Experimental, $X / D=0.7$

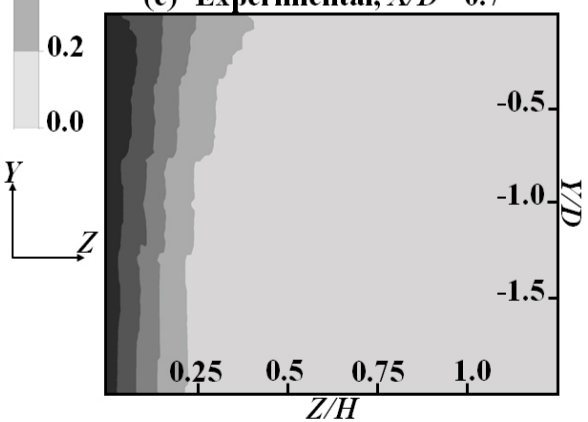

(g)- Experimental, $X / D=0.85$

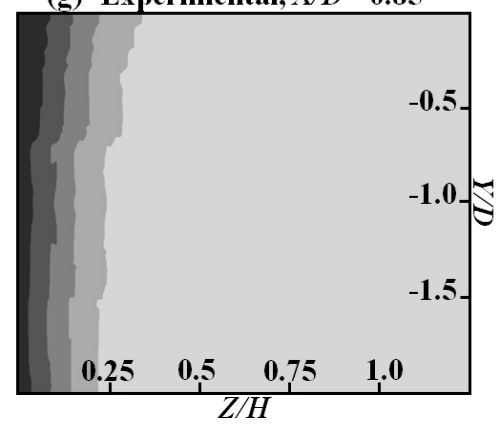

(b)- Numerical, $X / D=\mathbf{0}$

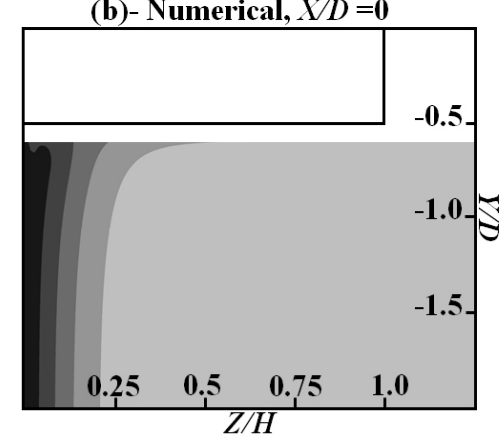

(d)- Numerical, $X / D=0.6$

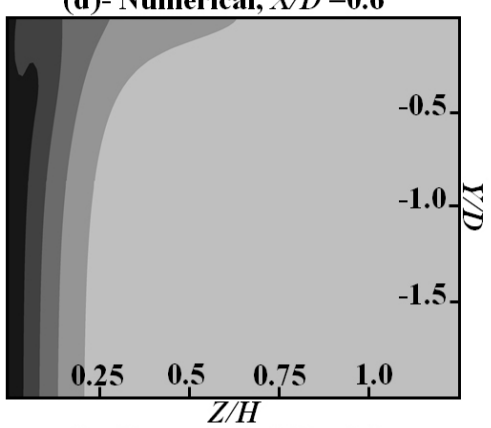

(f)- Numerical, $X / D=0.7$

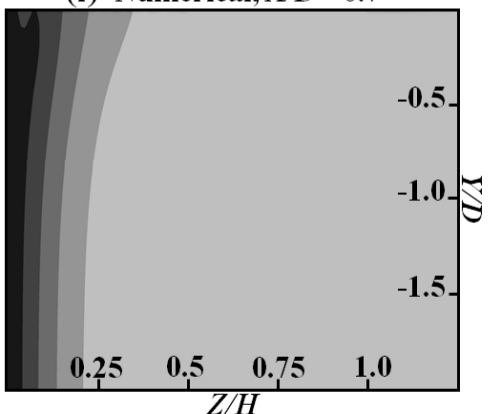

(h)- Numerical, $X / D=\mathbf{0 . 8 5}$

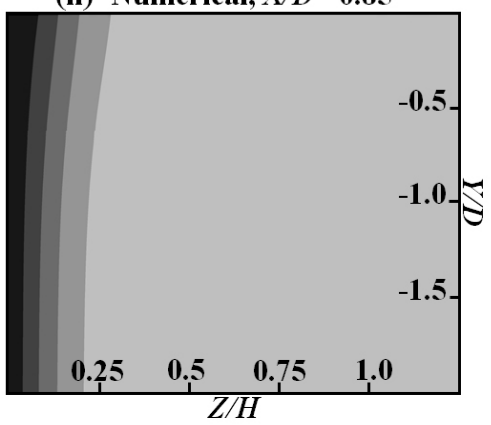

Fig. 2. The dimensionless temperature fields: (a) - experiment for $X / D=0$, (b) - numeric for $X / D=0,(\mathrm{c})$ - experiment for $X / D=0.6$, (d) - numeric for $X / D=0.6$, (e) - experiment for $X / D=0.7$, (f) - numeric for $X / D=0.7$, (g) - experiment for $X / D=0.85$, (h) - numeric for $X / D=0.85$

form a non-uniform density field. The arisen buoyant force encounters leading edge of circular cylinder. In the upstream region of the cylinder, the temperature of approaching flow to the leading edge of cylinder is very small except in the formed boundary layer very closed to the 
cylinder surface. It is due to this fact that diffusion arisen away from cylinder surface is less than approaching advection toward the cylinder [11]. When heated fluid approaches the leading edge of cylinder, at stagnation point the temperature distribution is divided into hot and cold regions by an imaginary line because of higher pressure at this point compared with adjacent regions. The hot flow moves toward the vertical plate, recirculates at junction of cylinder and heated plate, and leads to form horseshoe vortex system. For symmetry $Y Z$ plane $(X / D=0)$, the core of horseshoe vortex structure, which is located near the cylinder junction, can be seen in Fig. 2. In this work, as result of time-based solution, vortex structure is formed near the cylinder junction with elapse of time, and then a steady state condition will prevail along solution time so the center of the vortex stays at a certain position in accordance with flow recirculation.

In order to have better understanding of the temperature field in Fig. 2, the dimensionless temperature profiles in the symmetry plane $(X / D=0)$ for both numerical and experimental studies have been plotted in Fig. 3a at the different $Y$ coordinate below the leading edge of cylinder.

Fig. 3a displays the role of free convection flow approaching to the cylinder in modifying the flow field in the upstream region of the cylinder. The agreement between experimental and numerical results is quantitatively and qualitatively very good, especially in the thermal boundary layer region. The temperature distributions in the near wall domain and in the cylinder junction, where flow recirculates compare favorably. However, by moving away from cylinder in upstream region in $Y$ direction the consistency of experimental and numerical results get much closer. This is due to cylinder junction effects in the experiment where different disturbances appears in computational ambient compare to numerical simulation which simplifies the ambient parameters to resolve problem.

In order to demonstrate the effects of cylinder emplacement on approaching free convection flow, in Fig. 3b have been drawn experimental and numerical results of dimensionless temperature profiles for different $Y Z$ plane $(X / D=0.6,0.7,0.85)$. These graphs show dimensionless temperature at the angular coordinate equal to 90 degree relative to the leading edge of cylinder $(Y / D=0)$. Here, the angular coordinate zero is measured from the lower symmetry line of the cylinder (leading edge of cylinder). In Fig. 3b, analyzing the dimensionless temperature profiles on the heated vertical plate reveals the thermal boundary layer thickness, which in the upstream region of cylinder (angular coordinate between zero to 90 degree) is fairly uniform and approximately equals to $0.45 \mathrm{H}$. As shown in Fig. 3b, the effect of cylinder emplacement disappears by moving away from the cylinder in $X$ direction. In addition, the numerical results are in good compatibility with the experimental data.

As mentioned above, a part of flow, which separate at stagnation point on the leading edge of cylinder toward the vertical plate, recirculates at junction region, and leads to form horseshoe vortex system in the upstream of the cylinder. The arisen horseshoe vortex contributes intensive interaction of cold and hot flows, which undoubtedly have significant effect on free convective heat transfer in junction region [12]. By considering equation (1) for thermal energy, the heat transfer coefficient $(\alpha)$ is determined as ratio of the heat flux $(q)$ and the temperature difference between heated vertical plate and surrounding medium $\left(T_{W}-T_{\infty}\right)$ :

$$
\alpha=\frac{q}{T_{W}-T_{\infty}} .
$$

For the numerical solution, the values of free convection heat transfer coefficient are computed directly from numerical simulation based on finite volume method and equation (3). In experimental investigation, based on the collected data, the values of the heat transfer coefficient 
(a)

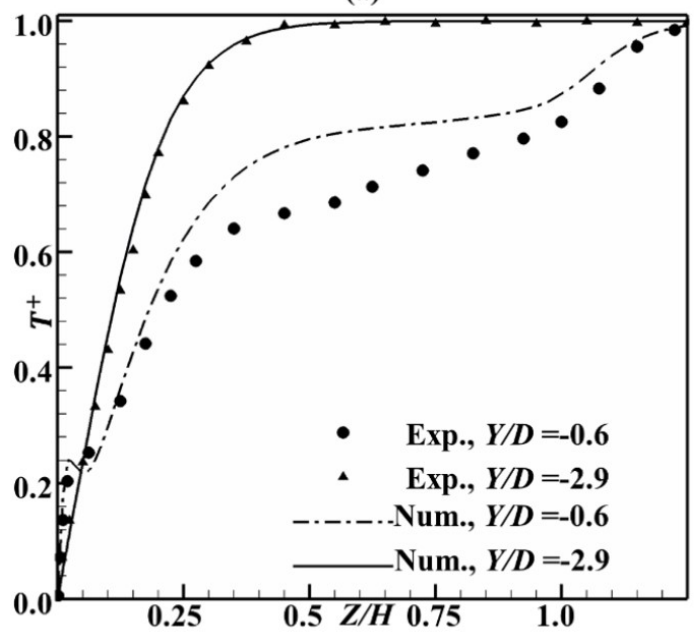

(b)

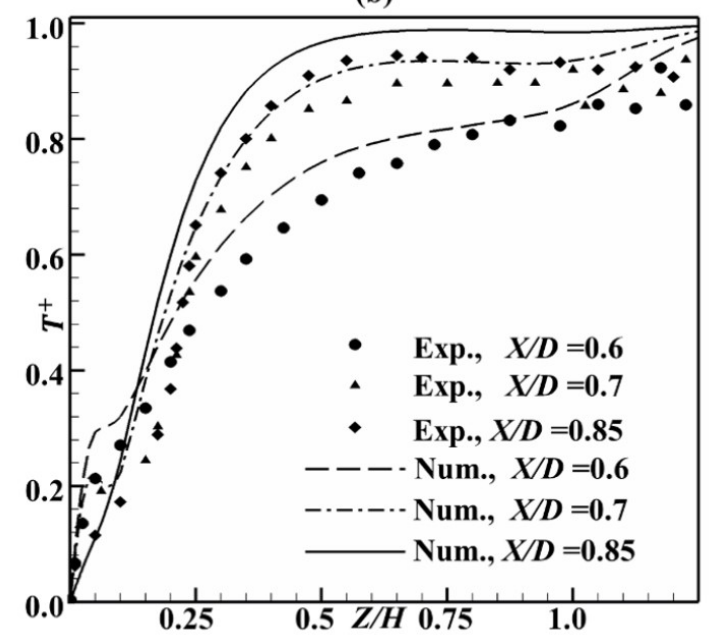

Fig. 3. The dimensionless temperature profiles; (a) - in the symmetry plane $(X / D=0)$, (b) - at the angular coordinate equal to 90 degree $(Y / D=0)$

are calculated by using equation (3). To aim this purpose, a thermal layer on the vertical plate is considered, on which the heat transfer is only due to conduction. Thus, the rate of convection heat transfer of ambient in the arisen convective layer on the heated vertical plate assumed equal to the rate of conduction heat transfer in imaginary conductive sub-layer. Therefore, the convection heat transfer is defined as follows:

$$
\alpha=\frac{\lambda}{T_{W}-T_{\infty}} \frac{\partial T}{\partial z}
$$

In equation (4), $\lambda$ is the thermal conductivity of ambient flow, which in this case considered constant and equals to $0.0242\left[\mathrm{Wm}^{-1} \mathrm{~K}^{-1}\right]$. The surrounding medium temperature $\left(T_{\infty}\right)$ was probed for each point during the measurement of computational ambient temperature. The value of the vertical plate temperature $\left(T_{W}\right)$ was obtained by linearization of temperature profile for desired point. Lastly, the value of temperature derivative relatively to the $Z$ coordinate (normal direction to the plate) was approximated by the line slope of temperature profile linearization in the vicinity of vertical plate with in conductive sub-layer. The existence of horseshoe vortex system in the junction region increase the convection heat transfer rate and subsequently leads to a thinner conductive sub-layer around cylinder, which may be considered during the temperature profile linearization.

Comparison of the numerical calculation of free convective heat transfer coefficient at different $Y Z$ plane with experimental results is shown in Fig. 4. For graphs within Fig. 4, the values of heat transfer coefficient meet a minimum at the junction of cylinder and vertical plate, which is described by existence of horseshoe vortex system and its configuration. As demonstrated in Fig. 4, the heat transfer rate near the cylinder surface is intensively more than other regions. This phenomenon can be explained by temperature differences between arisen thermal boundary layer and heated vertical plate. The bigger buoyancy force formed around the cylinder leads to increase in flow rate in this region and consequently the free convection heat transfer coefficient increases obviously.

As shown in Fig. 4, for spatial coordinates more than $0.4 D$ relative to cylinder surface, the experimental values quantitatively agree very well with the numerical results. For spatial 


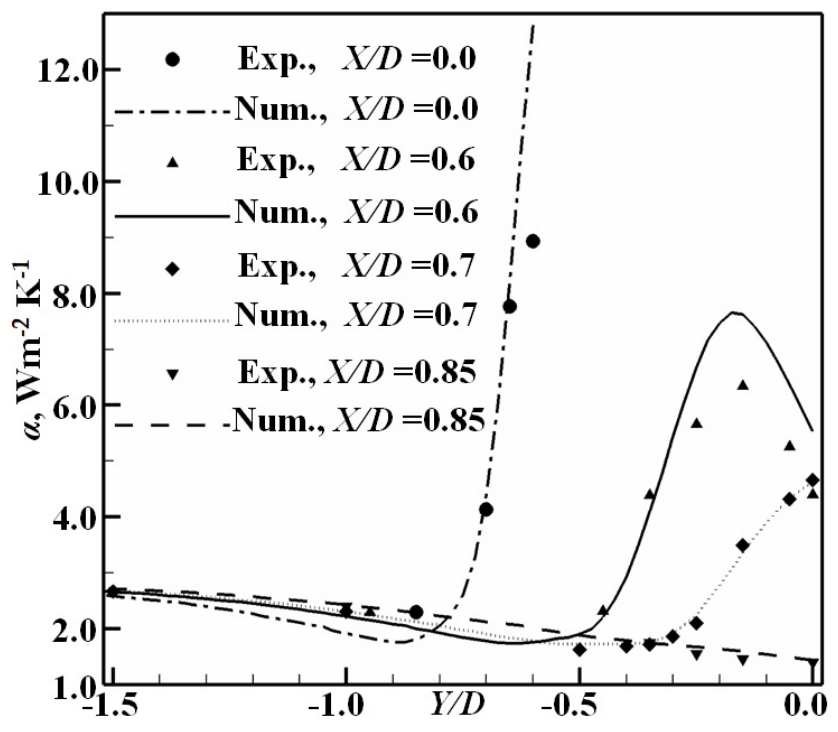

Fig. 4. The free convective heat transfer coefficient at different $Y Z$ plane

coordinates less than $0.4 D$ and more than $0.2 D$, the experimental results quantitatively and qualitatively are in acceptable agreement with numerical solution. Finally, for spatial coordinates less than $0.2 D$ (near cylinder surface), the experimental data only match with numerical calculations in configurative view. The maximum deviation of experimental heat transfer coefficients and its numerical values, which is seen in Fig. 4 around cylinder surface, is about $20 \%$. The appeared deviation between numerical and experimental results may be occurred due to disturbances existence near the junction of cylinder in experimental measurement, which were simplified to compute flow parameters in numerical solution. In addition, the accuracy of experimental procedure and devices, which can be affected by arisen errors during experiments, leads to accumulate deviation. The deviation can be categorized as researcher's errors and measurement errors, which consist of random errors and systematic errors. The random errors are due to real situation during the process of measurement and the systematic errors are because of specific experimental procedure. The statistical methods can foreshow the random errors and the calibration of devices can reduce the systematic errors. These errors must be analyzed to minimize the uncertainty of the obtained results. In this study, in order to analysis uncertainty of experimental data, we determine the parameters affecting the computation of heat transfer coefficient in equation (4). In this work, uncertainty of obtained values is due to measurement accuracy of surrounding medium temperature, vertical plate temperature and the rate of free convection heat transfer. These three parameters are considered as independent variables, so the uncertainty of heat transfer coefficient is defined as follows, where errors are neglected on length measurements [13]:

$$
\omega_{\alpha}= \pm\left[\left(\frac{\partial \alpha}{\partial q} \omega_{q}\right)^{2}+\left(\frac{\partial \alpha}{\partial T_{W}} \omega_{T_{W}}\right)^{2}+\left(\frac{\partial \alpha}{\partial T_{\infty}} \omega_{T_{\infty}}\right)^{2}\right]^{\frac{1}{2}} .
$$

It should be noted that the conduction and radiation heat transfer are neglected due to assuming pure buoyant flow on heated vertical plate. Therefore, the uncertainty of free convection 
heat transfer coefficient per unit area is determined as:

$$
\omega_{\alpha}= \pm\left[\left(\frac{1}{T_{W}-T_{\infty}} \omega_{q_{c o n v}}\right)^{2}+\left(\frac{-q_{c o n v}}{\left(T_{W}-T_{\infty}\right)^{2}} \omega_{T_{W}}\right)^{2}+\left(\frac{q_{c o n v}}{\left(T_{W}-T_{\infty}\right)^{2}} \omega_{T_{\infty}}\right)^{2}\right]^{\frac{1}{2}} .
$$

From equation (6), the uncertainty of heat transfer coefficient for different points, which considered in experimental study, varies between $2.96 \%$ and $7.41 \%$. From uncertainty analysis, it is found out that the maximum value of uncertainty for heat transfer coefficient is associated to near cylinder junction zone in symmetry plane $(X / D=0)$, where the disturbances expect to be maximum due to forming the core of horseshoe vortex system in this region. However, the uncertainty in order less than $10 \%$ is common. The high range of uncertainty about $10 \%$ appears from variation of the flow physical properties affected by temperature. Thus, in this work, the accuracy of the gathered experimental data are acceptable and satisfactory.

\section{Conclusion}

In this paper, the results of computational fluid dynamics simulation of free convective flow around circular cylinder, mounted on heated vertical plate in comparison with experimental case measurements are presented to understand the effect of cylinder junction on heat transfer rate in the upstream region of the cylinder. As result of experimental data gathering and non-stationary numerical solution, the following conclusions can be drawn:

- In the case of numerical study, by performing time-based simulation using Boussinesq approximation to resolve governing equation, a steady state solution was obtained around the junction area.

- By comparing the numerical and experimental results of dimensionless temperature and heat transfer coefficient, the validity of computational approach, its configurations and applied simplifications are approved.

- The computed values of heat transfer coefficient, demonstrate the considerable effects of cylinder emplacement on the free convection heat transfer rate. The heat transfer coefficient experiences its maximum at the symmetry plane of computational domain $(X / D=0)$ around the cylinder junction.

- The significant role of horseshoe vortex system on heat transfer calculation $[6,8,13]$ is illustrated partially and therefore is confirmed.

- By analyzing of heat transfer rate, this fact is emphasized that the high aspect ratio cylinder leads to enhance heat transfer coefficient in the upstream region of cylinder, as well as low aspect ratio cylinder $[2,8]$. Therefore, the increase of heat transfer coefficient in this region does not depend on cylinder height.

- In the vicinity region of the cylinder, flow is more complicated and the reliability of experimental study is decreased. In addition, associated uncertainty in this area is increased. It is occurred due to empirical measurement method sensitivity and arisen disturbances in reality. 
- In the junction region of cylinder, for spatial coordinates less than $0.2 D$, the results show that the compatibility of numerical solution and experimental data is reduced. Although the trend line of obtained results is matched each other favorably.

To sum up, by considering the limitations of experiment facility and numerical method deviations, it is appreciated to state that the accuracy of the obtained numerical and experimental results are satisfactory, acceptable and in good agreement. Furthermore, the used experimental and numerical approaches are valid to compute and analyze the characteristics of buoyancyinduced flow around the junction of horizontal cylinder, which located on the heated vertical plate.

\section{References}

[1] R.L.Simpson, Junction flows, Annu. Rev. Fluid Mech., 33(2001), 415-443.

[2] Y.S.Chumakov, A.M.Levchenya, H.Malah, The vortex structure formation around a circular cylinder placed on a vertical heated plate, J. Phys. Math.: St. Petersburg State Polytechnical University, 11(1)(2018), 56-66. DOI: 10.18721/JPM.11108

[3] H.Bhowmik, A.Faisal, A.Al-Yaarubi, N.Al-Alawi, Analyses of Natural Convection Heat Transfer from a Heated Cylinder Mounted in Vertical Duct, International Journal of Mechanical and Mechatronics Engineering, 12(3)(2018), 213-218.

[4] M.El-Amrani, M.Seaid, Numerical simulation of natural and mixed convection flows by Galerkin-characteristic method, Int. J. Numer. Meth. Fluids, 53(2007), 1819-1845.

[5] S.M.Elsherbiny, M.A.Teamah, A.R.Moussa, Natural convection heat transfer from an isothermal horizontal square cylinder, Alexandria Engineering Journal, 56(2017), 181-187.

[6] C.E.Clifford, M.L.Kimber, Optimizing laminar natural convection for a heat generating cylinder in a channel, Journal of Heat Transfer, 136(2014), no. 112502.

[7] T.J.Praisner, C.R.Smith, The dynamics of the horseshoe vortex and associated endwall heat transfer-Part I: Temporal behavior, Journal of Turbomachinery, 128(2006), 747-754.

[8] G.Polidori, J.Padet, Journal of Flow Visualization \& Image Processing, 10(2003), no. 1, 13-26. DOI: 10.1615/JFlowVisImageProc.v10.i12.20

[9] ANSYS Academic Research Mechanical, Release 16.2, Help System, Fluent Theory Guide, ANSYS, Inc. 2015.

[10] S.B.Koleshko, Y.S.Chumakov, Turbulent natural-convection heat transfer on a vertical surface under stepwise heating with inversion of heat flux, High Temp., 48(2010), 397-401 (in Russian).

[11] D.M.Christopher, B.X.Wang, Natural convection around a horizontal cylinder in a fluidsaturated porous media using Fourier series, Transactions on Engineering Sciences, 5(1994), $4-11$.

[12] H.Malah, Y.S.Chumakov, A.M.Levchenya, A study of the vortex structures around circular cylinder mounted on vertical heated plate, AIP Conference Proceedings, 1959(2018), no. 1, 050018. 
[13] S.O.Atayilmaz, Experimental and numerical study of natural convection heat transfer from horizontal concentric cylinders, International Journal of Thermal Sciences, 50(2011), $1472-1483$

\title{
Экспериментальное и численное исследование свободно-конвективного теплообмена в окрестности стика кругового цилиндра с вертикальной нагретой поверхностью
}

\author{
Хамид Малах \\ Юрий С. Чумаков \\ Санкт-Петербургский политехнический университет Петра Великого \\ Санкт-Петербург, Российская Федерация
}

\begin{abstract}
Аннотация. В данной работе исследуется влияние круглого цилиндра на трехмерные характеристики свободно-конвективного теплообмена. Круглый цилиндр установлен горизонтально на нагретой вертикальной поверхности и классифицируется как препятствие с высоким соотношением сторон, что означает, что высота цилиндра значительно больше чем его диаметр. Полученые результаты приведены для режима ламинарного потока. Кроме того, в ходе численного исследования определяющие дифференциальные уравнения решаются вокруг числа Грасгофа, равного $3 \times 10^{8}$. Чтобы определить области высоких градиентов температуры, температура потока показана в виде безразмерных контуров и диаграмм. В ближней области цилиндра вверх по потоку с помощью описания коэффициентов теплообмена, представленных градиентам температуры в заданных точках, изучается влияние расположения цилиндра на скорость теплопередачи. Как и ожидалось, значение коэффициента теплообмена, вызванного плавучестью, увеличивается в области соединения цилиндра и поверхности вверх по потоку. Максимальное значение коэффициента теплообмена появилось на плоскости симметрии исследуемой области, что соответствует расположению центра подковообразной структуры. В итоге обоснованность и достоверность численного и экспериментального подходов подтверждается путем вычисления девиации между числовыми и экспериментальными результатами, а также с помощью анализа неопределенности экспериментального метода.
\end{abstract}

Ключевые слова: поток, проходящий через стыки, свободно-конвективный теплообмен, ламинарный пограничный слой, коэффициент теплообмена. 